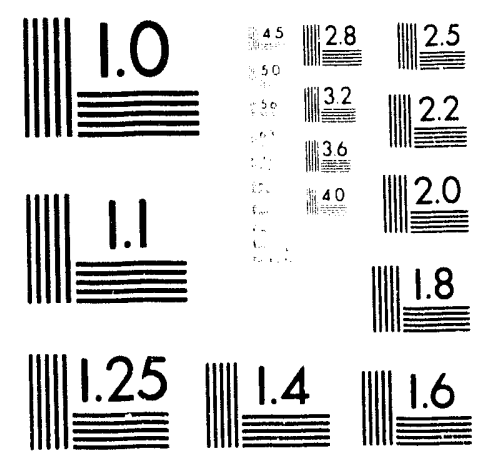



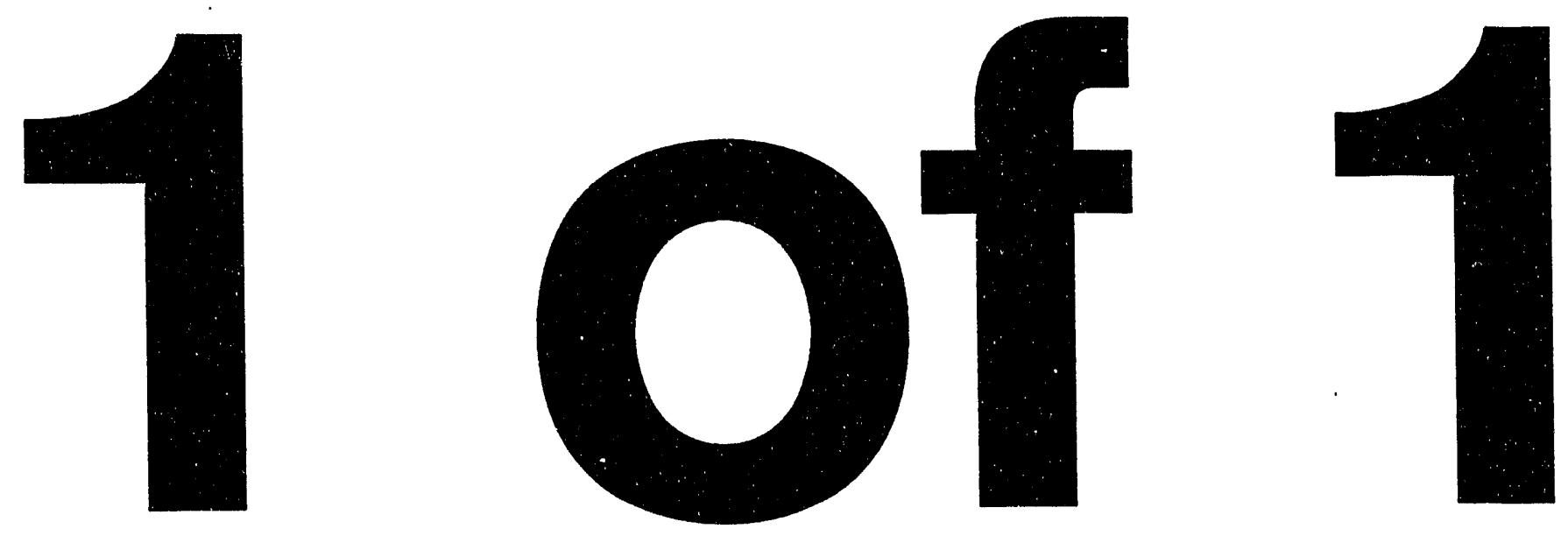


\title{
Analysis of DSP-Based Longitudinal Feedback System: Trials at SPEAR and ALS*
}

\author{
H. Hindi, N. Eisen, J. Fox, I. Linscott, G. Oxoby, L. Sapozhnikov \\ Stanford Linear Accelerator Center, Stanford University, Stanford, CA 94309 \\ and \\ M. Serio, INFN Laboratori Nazionali, Frascati, Italy
}

\begin{abstract}
Recently a single-channel prototype of the proposed PEPII longitudinal feedback system was successfully demonstrated at SPEAR and ALS on single-bunch beams. The phase oscillations are detected via a wide-band pick up. The feedback signal is then computed using a digital signal processo: (DSP) and applied to the beam by phase modulating the rf. We analyze results in the frequency-and the time-domain and show how the closed-loop transfer functions can be obtained rigorously by proper modeling of the various components of this hybrid continuous/digital system.
\end{abstract}

The technique of downsampling was used in the experiments to reduce the number of computations and allowed the use of the same digital hardware on both machines.

\section{INTRODUCTION}

It has been proposed that the longitudinal synchrotron oscillations in storage rings can be supressed using a DSPbased bunch-by-bunch feedback system [1]. In the bunchby-bunch approach, each bunch is treated as an individual oscillator driven by an unknown distırbance. The phase of each bunch is detected, a feedback signal particular to that bunch is computed using a digital signal processor, and is applied to that bunch on the following turn. The idea is that since this approach deals with each bunch on an individual basis, it can be extended to the multibunch case. The coupling would then be lumped into the unknown driving term. This technique would work if the coupling between the bunches is sufficiently weak. The programmable nature of the DSP-based feedback system and the technique of downsampling makes it possible to use the same digital hardware on different machines.

A single-channel prototype of this system was demonstrated successfully at SPEAR and, more recently, at ALS on single-bunch beams. We present some of the results of these experiments and show how they can be rigorously analyzed by appropriate modelling of the different components in the feedback system.

\section{EXPERIMENTAL SETUP}

The basic experimental setup used on both machines is shown in Figure 1. Since no wide-band kicker was available, the feedback was applied to the beam by phase modulating

\footnotetext{
-Work supported in part by U.S. Department of Energy contract DE-AC03-76SFO0515.

Presented at the Particle Acceterdtor Conference
}

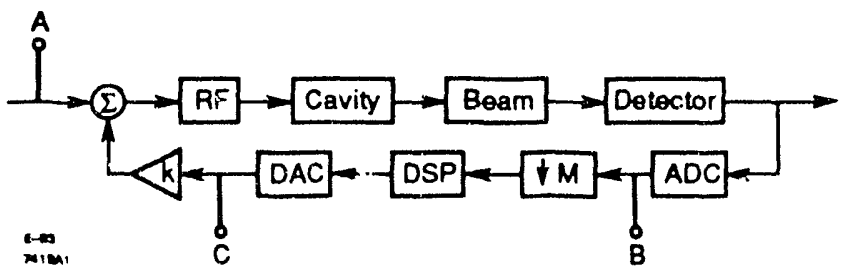

Figure 1: Experimental setup used at SPEAR and ALS.

the rf. A compensator (not shown) was included before the rf cavity to extend its bandwidth to beyond the frequency range over which the beam dynamics are interesting. The component $k$ represents an attenuator that was used to vary the loop gain. The $\downarrow \mathbf{M}$ represents downsampling ${ }^{1}$. This process had very little effect on the experiment as a whole.

Points A, B, and C represent poirts in the system between which transfer function measurements were made.

\section{MODEL OF SINGLE-BUNCH BEAM WITH FEEDBACK}

In this section we obtain theoretical expressions for the transfer functions from points $\mathrm{A}$ to $\mathrm{B},{ }^{2} T_{A \rightarrow B}(s)$, and from $\mathrm{B}$ to $\mathrm{C}, T_{B \rightarrow C}(s)$. From these expressions, the closed-loop transfer functionis obtained. Due to the large number of components in the loop, the modelling of delays plays an important role.

A. Model of the Beam

We model the beam phase oscillations, $\tau$, with respect to the rf as obeying the simple harmonic oscillator equation [4], except that we modify this equation to allow for a delay, $T_{d 1}$, in the response:

$$
\ddot{\tau}+2 \omega_{0} \zeta_{o} \tau+\omega_{o}^{2} \tau=-A u\left(t-T_{d 1}\right)
$$

where $\omega_{0}$ is the synchrotron frequency, $\zeta_{0}$ is the damping term, $A$ is a gain constant, and $u\left(t-T_{d 1}\right)$ is the driving in put to the system, delayed by $T_{d 1}$. These parameters can be easily extracted from the plots of the open-loop transfer functions of the system. Laplace transforming equation(1) yields the open-loop beam transfer function:

$$
B(s) \triangleq \frac{\tau(s)}{u(s)}=\frac{-A e^{-s T_{d 1}}}{s^{2}+2 \omega_{o} \zeta_{o} s+\omega_{o}^{2}}
$$

\footnotetext{
${ }^{1}$ This was used to reduce the number of computations by allowing only one out of every $M$ data samples to get to the DSP, see [2]

2 "s" here denotes the Laplace frequency variable.

DISTRIBUTION OF THIS DOCUMENT IS UNLIMITED
} 
We assume that, apart from contributing to the delay $T_{d 1}$ and to the gain $A$, the frequency responses of all the other components in the branch A $\rightarrow$ B are "flat" over the range of frequencies where the beam dynamics are of interest. Hence we take $B(s)$ to be our model for this branch, i.e., $T_{A \rightarrow B}(s)=B(s)$.

B. Model of tile Feedback

The objective of the feedback is to measure $\tau$ and process it to produce a feedback signal $u_{f b}$ that damps thsynchrotron oscillations. Ideally, this could be done using differential feedback [3], that corresponds to filtering $\tau$ with a differentiator, $H(s)=-K_{\text {diff }} s$, where $K_{\text {diff } f}$ is a constant. However, ideal differentiators have the unfortunate property of amplifying high-frequency noise. Hence, the DSP was used to implement a finite impulse response (FIR) digital filter [5] that approximates a differentiator over a finite frequency range. The transfer function of the FIR filter is given by

$$
H(s)=K_{\circ} \sum_{n=1}^{N} h(n) e^{-s T, n}
$$

where $K_{0}$ is the gain of the filt $\mathrm{r},\{h(n)\}_{1}^{N}$ are the coefficients of the FIR filter, and $T_{s}$ is the sampling rate. The coefficients used at SPEAR and ALS were given by:

$$
h(n)=\sin \left(\frac{2 \pi n}{N}-\Delta\right) \quad ; 1 \leq n-N .
$$

$\Delta$ is an adjustable parameter which gives control over the phase response of $H(s)$. In this single-channel prototype, additional delays due to the hardware exist, so we modify $H(s)$ to allow for these:

$$
H(s)=K_{o}\left(\sum_{n=1}^{N} h(n) e^{-s T_{v} n}\right) e^{-s T_{d}} .
$$

Once again, assuming that apart from contributing to a delay $T_{d 2}$ and to the gain $K_{o}$, the frequency responses of all the other components in the branch $B \rightarrow C$ are flat we can take $T_{B \rightarrow C}(s)=H(s)$. The only unknown parameters here are $K_{o}$ and $T_{d 2}$. These are obtained from measurements of the transfer function $T_{B \rightarrow C}(s)$.

\section{Closed-Loop Response}

Through the modelling process above, we have reduced the complicated system of Figure 1 to that shown in Figure 2.

Finding the closed-loop beam transfer function, $T_{A \rightarrow B}^{c l}(s)$, is now trivial: it is simply given by

$$
T_{A \rightarrow B}^{c l}(s)=\frac{B(s)}{1+k H(s) B(s)} .
$$

\section{RESULTS AND DISCUSSION}

Since much more data was available from the trial at ALS than at SPEAR, we focus on those results here,

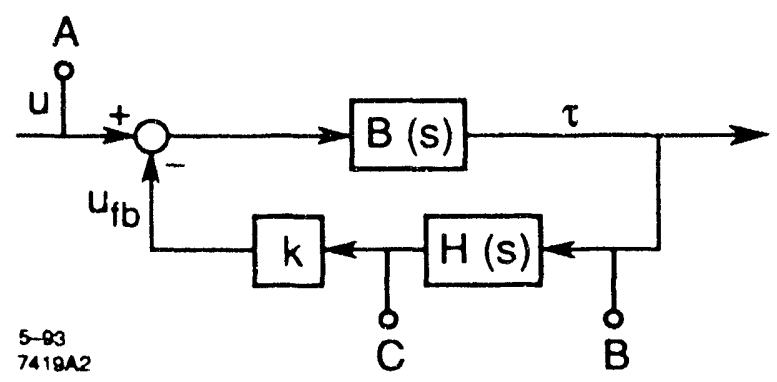

Figure 2: Model of the experimental setup used at SPEAR and AIS.
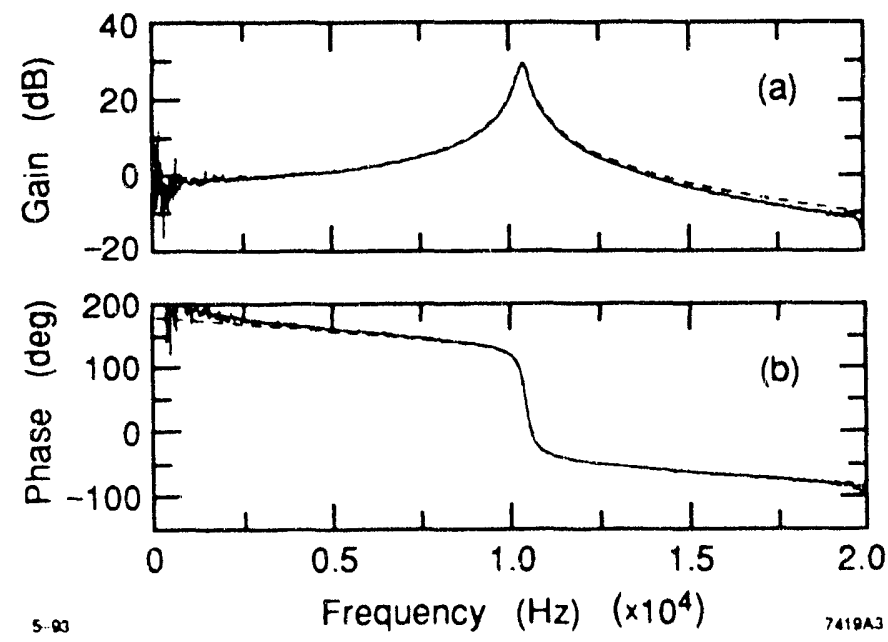

Figure 3: ALS measured versus fitted $T_{A \rightarrow B}(s)$.

though the resulis from both experiments were very similar. The ALS measured (solid) versus fitted (dashed) openloop beam and DSP transfer functions are showil in Figures 3 and 4 , respectively. In general, the agreement is good, except for the faster roll off of the measured responses. This roll off was probably due to the sample and hold of the DACs. The roll off at very low frequencies in the beam transfer function could have been the result of the response of any of the other components, whose frequency responses were assumed to be flat.

Figure 5 compares the ALS measured versus theoretical closed-loop responses, for several different loop gains. Notice that the damping (as measured by the width of the resonances) increases with loop gain for loop gains of -2 to $-19 \mathrm{~dB}$. However, at the larger loop gains of 24 and $29 \mathrm{~dB}$, the feedback actually began to drive new resonances at other frequencies. Thus we conclude that the closed-loop system using FIR feedback is conditionally stable, i.e., it is stable only over a finite range of loop gains. This means that there is actually a limit to the amount of damping that this type of feedback can provide.

Figure 6 shows the impulse responses corresponding to the loop gains above, obtained by inverse Fourier transforming the frequency responses above. As expected, the 


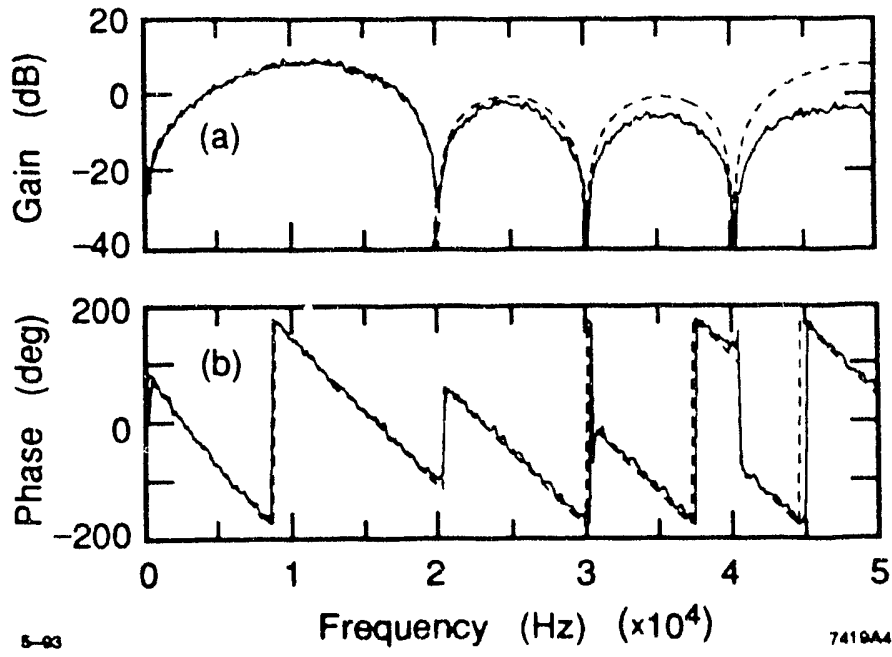

Figure 4: ALS measured versus fitted $T_{B \rightarrow C^{c}}(s)$. $N=6, \Delta=260^{\circ}$.

damping time constant is large for both very low and very high loop gains and is the shortest at 19dB (approximately two cycles), which is quite sufficient for accelerator physics purposes.

Despite their unusual appearance, these results were actually anticipated, as a result of an analysis similar to the one above.

In summary, we have presented an analysis of results from the trials of a single-channel feedback system on single-bunch beams at SPEAR and ALS. The results were analyzed by modelling each branch of the feedback system with a transfer function. 'The theoretical and measured closed-loop performance were in close agreement. Such a rigorous approach is necessary in the analysis, and more importantly, in the design of realistic feedback systems, such as the proposed PEP-II multi-bunch feedback system.

\section{ACKNOWLEDGEMENTS}

The authors would like to thank the ALS and SPEAR operations staff for their cooperation and enthusiasm during the machine physics runs, and Jean-Louis Pellegrin for his help and interest in the analysis.

\section{REFERENCES}

[1] "PEP-II, An Asymmetric B Factory-Design Update," H. Hindi, et al., Conceptual Design Report Update, SLAC 1992.

[2] H. Hindi et ai., "Downsampled Signal Processing for a B Factory Bunch-by-Bunch Feedback System," Proc. of the 1992 European Particle Accelerator Conf., p. 1067.

[3] "Feedback Control of Dynamic Systems," G. Franklin, Addison-Wesley, 1991.

[4] "The Physics of Electron Storage Rings, An Introduction," M. Sands, SLAC-121 U-28, 1970.

[5] "Discrete-Time Signal Processing," A. V. Oppenheim and R. W. Schafer, Prentice-Hall, 1989
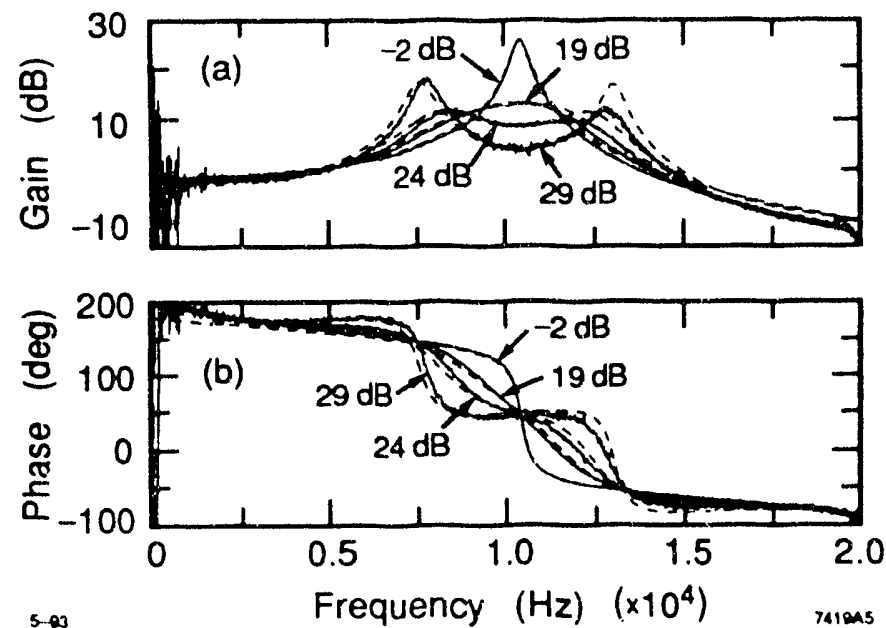

Figure 5: ALS measured versus fitted $T_{A \rightarrow B}^{c l}(s)$ for loop gains of $-2,19,24$ and $29 \mathrm{~dB}$.
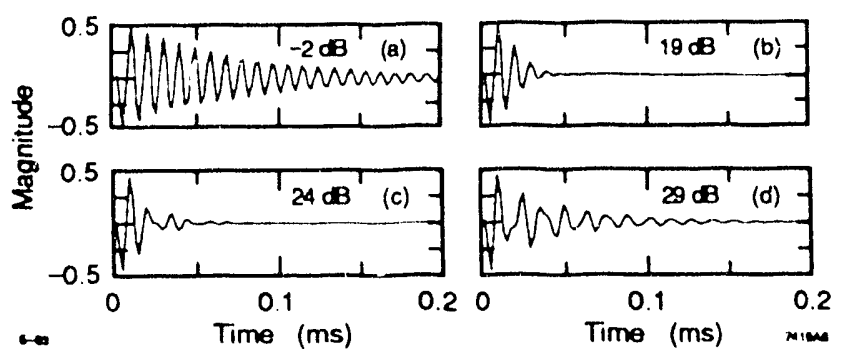

Figure 6: ALS closed-loop impulse responses for loop gains of $-2,19,24$ and $29 \mathrm{~dB}$. 


\section{DISCLAIMER}

This report was prepared as an account of work sponsored by an agency of the United States Government. Neither the United States Government nor any agency thereof, nor any of their employees, makes any warranty, express or implied, or assumes any legal liability or responsibility for the accuracy, completeness, or usefulness of any information, apparatus, product, or process disclosed, or represents that its use would not infringe privately owned rights. Reference herein to any specific commercial product, process, or service by trade name, trademark, manufacturer, or otherwise does not necessarily constitute or imply its endorsement, recommendation, or favoring by the United States Government or any agency thereof. The views and opinions of authors expressed herein do not necessarily state or reflect those of the United States Government or any agency thereof. 

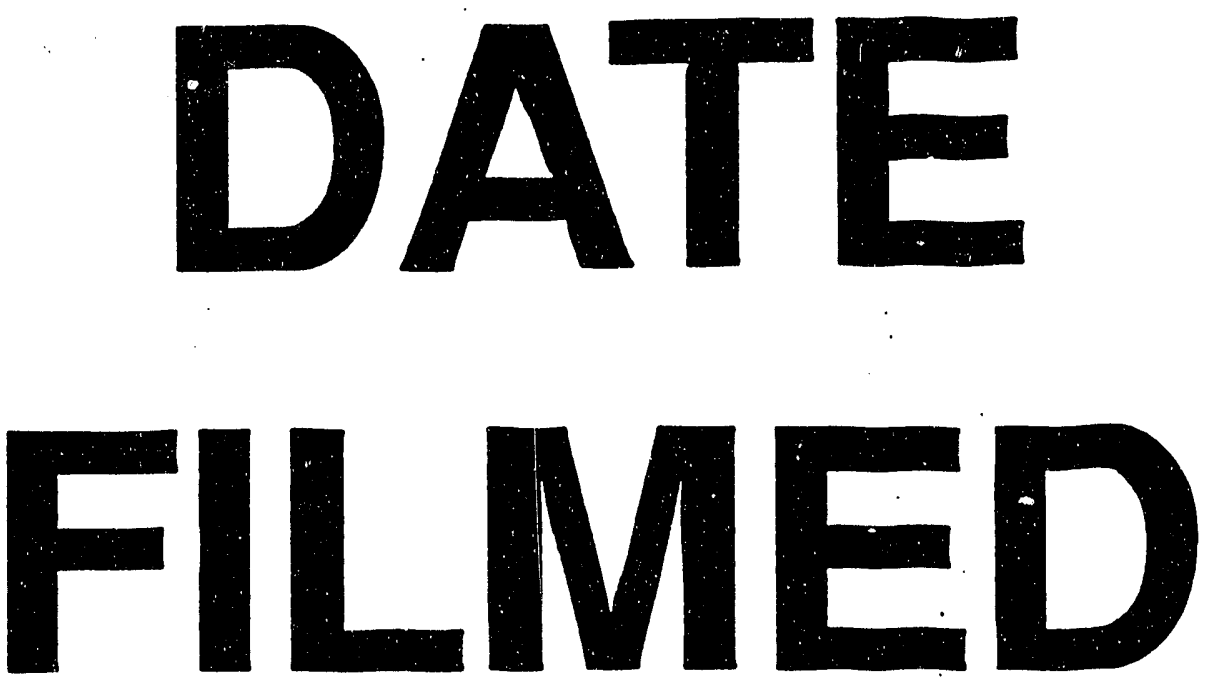

$11 / 19 / 93$
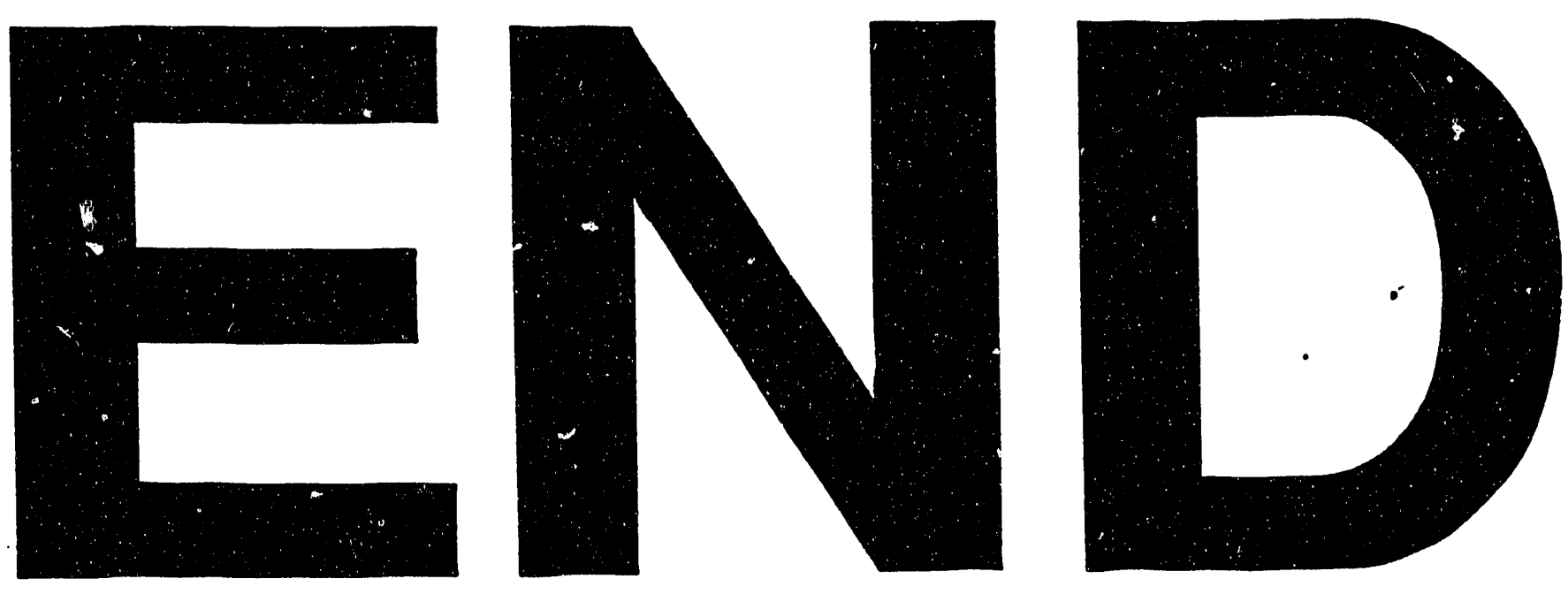
